\title{
Analysis of chromosome structure in Musaceae using oligo painting
}

Šimoníková D., Doležel J., Hřibová E.*

Institute of Experimental Botany, Centre of Plant Structural and Functional Genomics,

Olomouc, Czech Republic

*e-mail:hribova@ueb.cas.cz

The family Musaceae comprises genera Musa, Ensete and Musella. While Ensete $(2 n=18)$ and Musella $(2 n=18)$ are represented by a few and one endemic species, respectively, genus Musa contains about 70 different species. Based on plant morphology and basic chromosome number, Musa species are classified into four sections. The largest of them, Eumusa $(x=11)$ comprises most of edible banana cultivars. They originated by intra- and inter-specific crosses between wild diploids M. acuminata and M. balbisiana. The section Rhodochlamys $(x=11)$ contains ornamental species, which are closely related to those of Eumusa. The section Australimusa $(x=10)$ is represented by species growing in Southeast Asia and contains a peculiar group of edible banana clones known as Fe'i. The section Callimusa is the most diverse and contains species differing in basic chromosome number $(x=9,10)$ and species which seem to be closely related to Ensete and Musella. In order to analyze chromosome structure and karyotype evolution in Musaceae, we used the recently developed method of oligo painting FISH. Pools of chromosome arm-specific oligomers were designed using the reference genome sequence of double haploid M. acuminata 'DH Pahang'. The oligomers were labeled using reverse transcription either by hapten- or fluorescently-labeled primers and used for FISH in a set of accessions representing different subspecies of M. acuminata, in M. balbisiana, and their intra- and inter-specific hybrids. The oligo painting probes were also tested for their suitability to detect chromosome rearrangements in more distant species of the family Musaceae. The chromosome oligo painting enabled anchoring pseudomolecules to individual chromosomes, creation of molecular karyotypes and identification of large structural chromosome rearrangements. The method of oligo painting FISH thus opens avenues for comparative analysis of structural chromosome changes to shed light on karyotype evolution in Musaceae.

Acknowledgements: This work was supported by the Grant Agency of the Czech Republic (award No. 19-20303S). The computing was supported by the National Grid Infrastructure MetaCentrum (grant No. LM2010005 under the program Projects of Large Infrastructure for Research, Development, and Innovations). 